\title{
A Novel LMNA Mutation Causes Altered Nuclear Morphology and Symptoms of Familial Partial Lipodystrophy (Dunnigan Variety) with Progeroid Features
}

\author{
B. Saha ${ }^{a}$ D. Lessel ${ }^{c}$ F.M. Hisama ${ }^{b}$ D.F. Leistritz ${ }^{a} \quad$ K. Friedrich ${ }^{c} \quad$ G.M. Martin ${ }^{a}$ \\ C. Kubisch ${ }^{c}$ J. Oshima ${ }^{a}$ \\ a Department of Pathology, and bivision of Genetics, Department of Medicine, University of Washington, \\ Seattle, Wash., USA; ' Institute of Human Genetics, Center for Molecular Medicine Cologne, and Cologne \\ Excellence Cluster on Cellular Stress Responses in Aging-Associated Diseases (CECAD), University of Cologne, \\ Cologne, Germany
}

\section{Key Words}

Human · Lamin • Progeroid syndrome - Werner syndrome

\begin{abstract}
Dunnigan-type partial lipodystrophy (familial partial lipodystrophy, Dunnigan variety, FPLD2) can be caused by LMNA mutations. We identified a novel heterozygous $L M N A$ mutation, P485R, in a patient referred to the International Registry of Werner Syndrome because of features consistent with that of progeroid disorder but who was wild type at the WRN locus. The novel mutation is located 2 amino acids away from the canonical FPLD mutations in exon 8 of the LMNA gene. Immunocytochemical analysis revealed abnormal nuclear morphology characteristic of laminopathies within primary fibroblast cultures, but not in a lymphoblastoid cell line, in keeping with previous observations. Our findings indicate that FPLD2 should be considered in the differential diagnosis of the Werner syndrome.

Copyright $\odot 2010$ S. Karger AG, Basel
\end{abstract}

Familial partial lipodystrophy of the Dunnigan type (FPLD2, OMIM 151660) is characterized by the loss of subcutaneous fat tissue in upper and lower extremities as well as gluteal and truncal regions together with the accumulation of fat within tissues of the face and neck. These characteristic symptoms begin around puberty [Dunnigan et al., 1974; Garg, 2000]. FPLD2 patients frequently develop type 2 diabetes mellitus and hypertriglyceridemia in their 20s. They may also develop acanthosis nigricans, hirsutism and menstrual abnormalities [Garg, 2004; Vantyghem et al., 2008]. Clinical phenotypes are more pronounced in women, who exhibit higher frequencies of diabetes and atherosclerotic heart disease [Garg, 2000]. Because of the gradual loss of subcutaneous adipose tissue within arms and legs, these limbs appear to be unusually muscular [Garg, 2000].

FPLD2 can be caused by heterozygous mutations of the LMNA gene (OMIM 150330). The LMNA gene encodes the nuclear intermediate filaments, lamin A and C, generated by alternative splicing [Fisher et al., 1986]. Lamin A/C proteins consist of an N-terminal globular

\section{KARGER}

Fax +41613061234 E-Mail karger@karger.ch www.karger.com
Junko Oshima, $\mathrm{PhD}$

Department of Pathology, University of Washington

Box 357470, 1959 NE Pacific St.

Seattle, WA 98195-7470 (USA)

Tel. +1 206543 5088, Fax +1 206685 9256, E-Mail picard@ u.washington.edu 
domain, an $\alpha$-helical rod domain and a C-terminal globular domain. Lamin A, but not lamin $\mathrm{C}$, has a tail region at the C-terminal end that undergoes successive posttranslational modifications for the maturation from prelamim A to lamin A [Broers et al., 2006; Worman and Bonne, 2007]. Lamin A/C forms dimers through the $\alpha$ helical domains and is assembled with other structural proteins to form the nuclear lamina, which provide structural support for the inner nuclear membrane. In addition to these structural roles, lamin A/C interacts with chromatin and transcriptional factors and may therefore play an important role in gene regulation [Broers et al., 2006; Worman and Bonne, 2007].

Mutations of the LMNA gene result in a wide variety of disorders, including muscular dystrophies, cardiomyopathies, lipodystrophies, and progeroid syndromes [Broers et al., 2006; Worman and Bonne, 2007]. Although phenotypic overlaps have been widely recognized, there are recognizable genotype-phenotype relationships [Hegele, 2005; Hegele and Oshima, 2007].

The first identified FPLD mutations of the LMNA gene were heterozygous missense substitutions at amino acid 482, Arg482Gln [Cao and Hegele, 2000], Arg482Trp and Arg482Leu [Shackleton et al., 2000]. Subsequently, Gly465Asp was also identified as a FPLD mutation [Speckman et al., 2000]. These mutations were located in exon 8 of the $L M N A$ gene, corresponding to the C-terminal globular region of the lamin A and C proteins. FPLD features with overlapping symptoms or atypical presentations have been attributed to other heterozygous LMNA mutations, including Arg582His [Speckman et al., 2000], Arg60Gly [van der Kooi et al., 2002], Arg28Trp, Arg62Gly, Arg419Cys, Leu515Glu [Haque et al., 2003], Asp230Asn, Arg399Cys, Ser573Leu [Lanktree et al., 2007], and Asp192Val [Subramanyam et al., 2009]. Overall, approximately $85 \%$ of FPLD2 patients are thought to carry one of the heterozygous mutations at Arg482 [Broers et al., 2006].

The hallmark of cells harboring LMNA disease mutations is the nuclear morphological abnormality described as nuclear blebbing [Broers et al., 2006; Worman and Bonne, 2007]. Primary fibroblasts carrying heterozygous FPLD2 mutations, Arg482Gln or Arg482Trp, were shown to exhibit various degrees of nuclear envelope disorganization [Vigouroux et al., 2001].

In the present study, we report a novel LMNA mutation, Pro485Arg, located 3 amino acids away from the canonical FLPD2 mutations. We present cell biological evidence to support our conclusion that this mutation is responsible for the spectrum of phenotypes suggestive of both Werner syndrome and a lipodystrophic variant, FLPD2. Given these overlaps in clinical presentation, we suggest that FLPD2 be considered in the differential diagnosis of Werner syndrome.

\section{Materials and Methods}

\section{Patient Samples}

The patient was referred to the International Registry of Werner Syndrome, Department of Pathology, University of Washington, Seattle, Wash., USA, for mutational analysis of the WRN gene. Lymphoblastoid cell lines (LCLs) were established using Epstein Barr virus as described previously [Huang et al., 2006]. Control DNA samples were obtained through the National Long Term Care Survey (Department of Pathology, University of Washington), a population-based sample of US residents [Risques et al., 2010]. This study was approved by the Institutional Review Board of the University of Washington.

\section{DNA Sequencing}

Genomic DNA was isolated from LCLs. DNA sequencing of coding regions of $L M N A$ was performed as described previously [Chen et al., 2003]. Briefly, genomic PCR primers were designed to amplify all coding exons together with flanking intronic sequences (50-100 bp), based on the GenBank reference sequence, NG_008692.1. PCR products were sequenced using Big Dye terminator version 3.1 chemistry on an ABI $3730 \mathrm{XL}$ or ABI 310 Genetic Analyzer (Applied Biosystems, Foster City, Calif., USA). Sequences were analyzed with commercially available Mutation Survey software (SoftGenetics, State College, Pa., USA).

\section{Immunocytochemistry and Nuclear Morphology}

Immunofluorescence staining was carried out as described previously [Huang et al., 2005, 2008]. Primary fibroblasts from the propositus (VGS1010), a LMNA mutant strain carrying a R133L mutation (PORTU8010) and a control (82-6) were grown for $24 \mathrm{~h}$ on cover slips. Cells were then fixed in either $70 \%$ ethanol and $0.15 \mathrm{~mm}$ glycine at $-20^{\circ} \mathrm{C}$ or in $3 \%$ paraformaldehyde (PFA) in PBS ( $\mathrm{pH} 7.4$ ) at room temperature for $20 \mathrm{~min}$, followed by permeabilization with $1 \%$ Triton-X in PBS, then processed for immunofluorescence as follows: cells were incubated with anti-lamin A/C (clone 636) (sc-7292, Santa Cruz Biotechnology, Santa Cruz, Calif., USA) (1:100 dilution) for $30 \mathrm{~min}$ at $37^{\circ} \mathrm{C}$ followed by Alexa Fluor 594-conjugated goat anti-mouse antibody (1:200 dilution) (A-11005, Molecular Probes, Eugene, Oreg., USA) for 30 min at $37^{\circ} \mathrm{C}$. Cells were mounted onto slides using Vectashield (H-1000 Vector Laboratories, Burlingame, Calif., USA) with DAPI (for nuclear staining) and observed and photographed with a Nikon Upright (Nikon Eclipse E600) microscope at the Keck Center for Imaging, University of Washington.

To assess the degree of nuclear irregularity, nuclear contour ratios (circularity of nucleus, $4 \pi \times$ area/perimeter ${ }^{2}$ ) were determined in 100 randomly selected nuclei of control and LMNA mutant fibroblasts using MetaMorph software [Huang et al., 2005, 2008]. A nuclear contour ratio (NCR) with a value of 1 indicates a perfect circle, whereas values approaching 0 indicate increasing degrees of irregularity. Statistical significance was determined in 
2 ways - a t test of the differences in the means of NCR between cultures of different genotypes and a $\mathrm{c}$ test to determine the significance of the variances of the distributions of NCR values between cultures of different genotypes [Huang et al., 2005, 2008].

\section{Case Report}

The proband, Registry VGS1010, was a white female evaluated at age 12 years for short stature. Growth hormone and leutinizing hormone stimulation tests were normal. Her body habitus was slender, with a lack of subcutaneous tissue, and delayed puberty. She was treated with conjugated equine estrogen and oxandrolone. Regular menses commenced at age 16 years, after the cessation of hormonal treatment. An eye exam was normal. The clinical impression was Werner syndrome. She became pregnant at age 19 and had an elective termination. Her other medical history included asthma, anxiety, and a GI hemorrhage from an intestinal polyp. She was married, worked as an office assistant and was active in several sports.

Her family history was notable for a younger sister who appeared unaffected in photographs, a maternal uncle with mild intellectual impairment and another maternal uncle who died of leukemia in his 40 s. Her parents were nonconsanguineous and of mixed European background.

On physical exam at age 28 years, her height was $145.8 \mathrm{~cm}$ ( $<5$ th percentile), weight was $25.3 \mathrm{~kg}$ ( $<3 \mathrm{rd}$ percentile). Her BP was elevated at 220/140 mm mercury. She appeared older than her stated age and lacked subcutaneous adipose tissue. Her skin was thin with a 'peppered' hypopigmented appearance. The hair was thin but not prematurely gray. She had moderate proptosis, large ears, prominent nose with beaked nasal tip, and a recessed chin. Her voice was high-pitched. Cardiac exam showed a IV/VI systolic murmur. There was no organomegaly. A healed knee scar had a cigarette paper appearance. A neurological examination was unremarkable.

Laboratory tests showed normal fasting glucose, thyroid stimulating hormone, creatinine and a lipid panel. On echocardiogram, she was found to have a heavily calcified atrial valve with reflux, moderate aortic stenosis and calcific chordae. There was a thickened mitral valve with moderate mitral regurgitation and moderate aortic insufficiency. Photographs of the patient are not available.

\section{Results}

\section{Identification of a Novel LMNA Mutation}

Genomic sequencing of the WRN gene did not show any mutations. Western analysis of proteins extracted from the patient's LCL revealed a WRN protein of the expected size and amount when compared to that of the control. These findings (data not shown) ruled out a molecular diagnosis of Werner syndrome.

Genomic sequencing of the LMNA gene revealed a heterozygous alteration, c.1455A $>\mathrm{G}$ (Pro485Arg), in exon 8 (fig. la, c), within the globular tail domain of

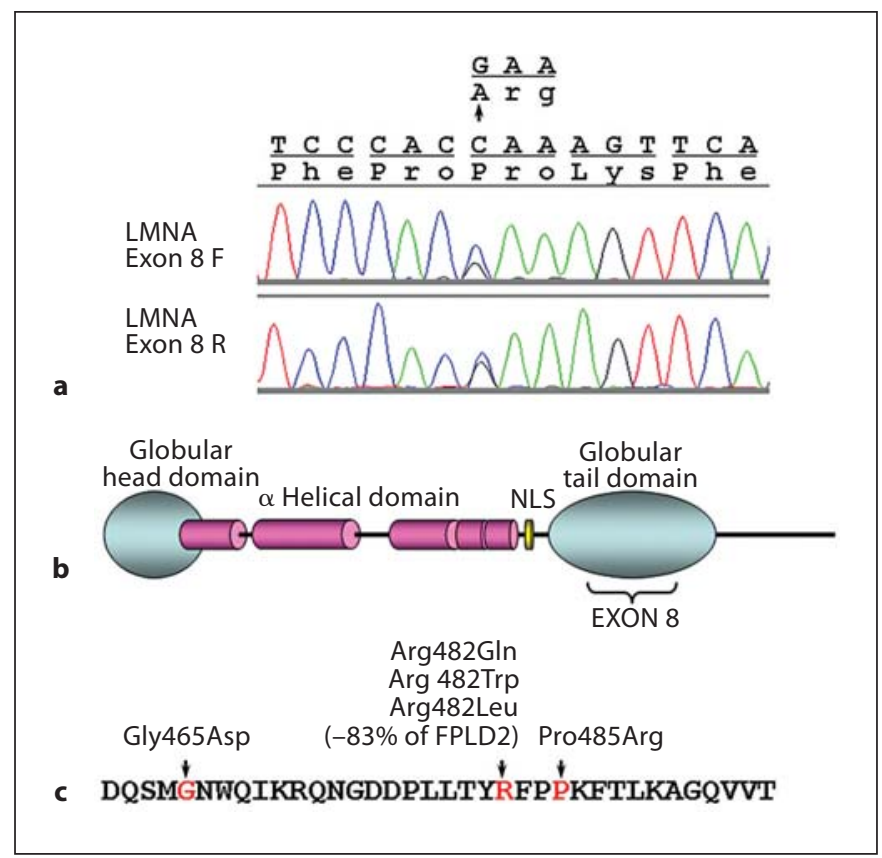

Fig. 1. FPLD2 mutations in $L M N A$ exon 8. a Detection of c.1455A >G (Pro485Arg) mutation. Chromatographs of LMNA exon 8 sequences are shown in forward (top) and reverse (bottom) directions. b Diagram of lamin A protein, showing the exon 8 region in globular tail domain. NLS = Nuclear localization signal. c Previously described FPLD2 mutation in LMNA exon 8. Only mutations reported to result in patients with typical FPLD2 phenotypes are indicated (amino acids coded by exon 8 ).

lamin A/C (fig. 1b). This alteration was not seen in the genomic DNA of 242 control subjects.

The Polyphen program, which predicted that the first identified FPLD mutation, Arg482Gln, was benign, also predicted Pro485Arg to be benign, suggesting that this prediction program is somewhat limited. While the LMNA gene has been extensively sequenced and more than 300 mutations are described in 2 gene specific databases, one at the Universal Mutation Database (www. umd.be/LMNA/) and the other at Leiden University (http://www.dmd.nl/nmdb/home.php?select_db=LMNA), our alteration was not listed, either as a pathogenic or benign variant. Pro485Arg was also not in the publicly available SNP database (http://www.ncbi.nlm.nih.gov/ projects/SNP). This suggests that a Pro485Arg change is an extremely rare event. Parental samples were unobtainable, and thus we could not determine whether the change was de novo or inherited. 
Fig. 2. Abnormal nuclear morphology and lamin $\mathrm{A} / \mathrm{C}$ staining patterns in primary fibroblasts with the p.P485R mutation. Control fibroblasts (82-6), LMNA mutant fibroblasts (Registry VGS1010 with the p.P485R mutation and PORTU8010 with the p.R133L mutation) were stained for lamin A/C and counterstained with DAPI. Notice the irregular thickening and aggregates of nuclear lamina in the nuclei of LMNA mutant fibroblasts.

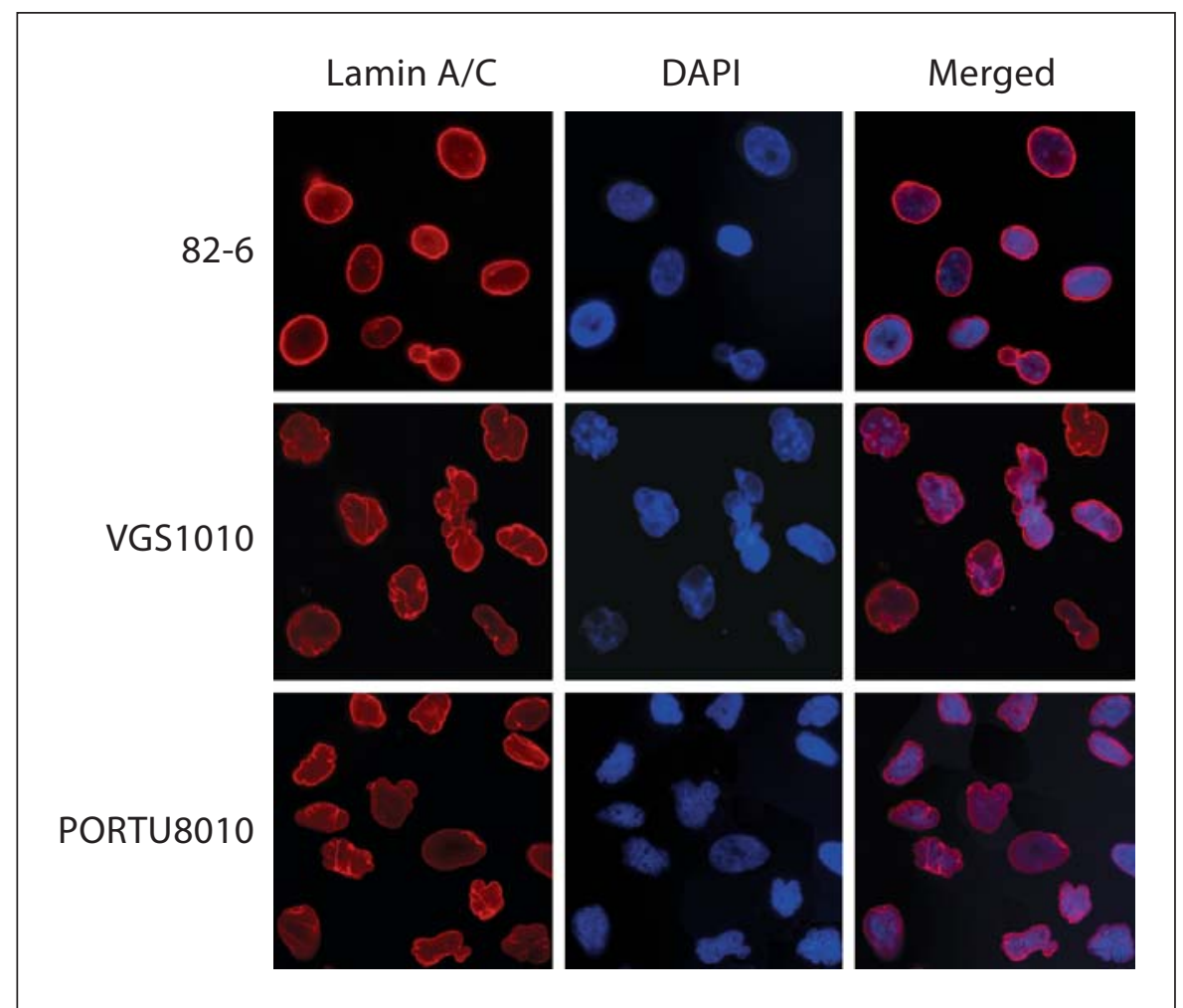

Table 1. Nuclear morphological abnormalities in primary fibroblasts with $L M N A$ mutations as compared to a normal control $(82-6, w t)$

\begin{tabular}{lllll}
\hline Cell line & $\begin{array}{l}\text { LMNA } \\
\text { mutation }\end{array}$ & $\begin{array}{l}\text { Nuclear } \\
\text { contour ratio }\end{array}$ & $\begin{array}{l}\mathrm{p} \\
\text { (t test) }\end{array}$ & $\begin{array}{l}\mathrm{p} \\
\text { (f test) }\end{array}$ \\
\hline $82-6$ & wt & $0.847 \pm 0.057$ & NA & NA \\
VGS1010 & Pro485Arg & $0.817 \pm 0.050$ & 0.017 & 0.635 \\
PORTU8010 & Arg133Leu & $0.788 \pm 0.100$ & $<0.0001$ & 0.031 \\
\hline
\end{tabular}

\section{Demonstration of Abnormal Nuclear Morphology in}

\section{LMNA Mutant Patient Fibroblasts}

Nuclear morphological abnormalities often described as 'blebbing', 'herniation' or 'denting' are characteristic cellular features of LMNA mutant cells. To investigate whether the Pro485Arg mutation resulted in similar abnormalities in cells from this patient, we performed immunofluorescence analysis with lamin A/C and DAPI staining in patient primary fibroblasts and compared the results to those of control fibroblasts, 82-6 and to fibroblasts bearing a known LMNA mutation, PORTU8010 with an Arg133Leu mutation [Chen et al., 2003] (fig. 2).
Control fibroblasts showed round or ovoid nuclei with only an occasional cell exhibiting blebbing. In contrast, a significant number of nuclei in the VGS1010 fibroblasts were observed with nuclear blebs and lobulation, similar to those in the PORTU8010 fibroblasts (fig. 2).

The degree of nuclear morphological abnormality was quantitated via nuclear contour ratios (NCR) calculated as $4 \pi \mathrm{A} / \mathrm{P}^{2}$ (table 1 ). The NCR represents how closely an object represents a circle. In control fibroblasts the contour ratio was $0.847 \pm 0.057$, whereas in the VGS1010 the contour ratio was $0.817 \pm 0.050(\mathrm{p}=0.017)$; for PORTU8010, the ratio was $0.788 \pm 0.100(\mathrm{p}<0.0001)$. These indicate that there was a statistically significant abnormality in the shape of the nuclei in our patient's fibroblasts. The variance of the distributions of NCR values from populations of LMNA mutant fibroblasts did not differ from that of the control ( $\mathrm{p}=0.635$ for VGS1010 and 0.031 for PORTU8010). This indicated that the dysmorphology phenotypes of LMNA mutant fibroblasts are comparatively uniform.

The NCR of LCLs derived from VGS1010 (0.767 \pm $0.164)$ and that of the control $(0.768 \pm 0.160)$ were not significantly different ( $\mathrm{t}$ test, $\mathrm{p}=0.943$ ), consistent with our previous experience that immortalization appears to 
Table 2. Clinical features of the patient with $L M N A$ P485R mutation and comparisons to Werner syndrome and Dunnigan type partial lipodystrophy

\begin{tabular}{llll}
\hline Symptoms & $\begin{array}{l}\text { This } \\
\text { patient }\end{array}$ & $\begin{array}{l}\text { Dun- } \\
\text { nigan }\end{array}$ & $\begin{array}{l}\text { Werner } \\
\text { syndrome }\end{array}$ \\
\hline Short stature (W) & + & - & + \\
Lack of pubertal growth spurt & + & - & + \\
Parental consanguinity (W) & - & - & + \\
Graying/thinning hair (W) & + & - & + \\
Skin atrophy, pigment changes (W) & + & - & + \\
Acanthosis nigricans, xanthomata & - & + & - \\
Cataracts by age 30 (W) & $?$ & - & + \\
High-pitched voice & + & - & + \\
Diabetes mellitus & - & + & + \\
Lipodystrophy (D) & + & + & \\
Hypertriglyceridemia (D) & - & + & \\
Hypogonadism & + & + & + \\
Atherosclerosis & - & + & + \\
Aortic valve calcification & + & - & - \\
Hypertension & + & + & - \\
Osteoporosis & $?$ & - & + \\
Leg ulcers & - & - & + \\
Muscle hypertrophy & - & + & - \\
Mesenchymal neoplasm & - & - & + \\
Intestinal polyp & + & - & - \\
\hline & & &
\end{tabular}

$\mathrm{W}=$ Cardinal feature of Werner syndrome; $\mathrm{D}=$ cardinal feature of Dunnigan familial lipodystrophy.

mask the nuclear morphological abnormality [Nguyen et al., 2007].

Control fibroblasts showed uniform distribution of lamin $\mathrm{A} / \mathrm{C}$ along the peripheries of the nuclei, whereas nuclei in the VGS1010 fibroblasts displayed lamin A/C aggregates at the peripheries. There were also 'fold'-like structures in the nucleoplasm of PORTU8010 (fig. 2). These structures may represent focal twists of the nuclear envelope [Vigouroux et al., 2001].

\section{Discussion}

In this study, we report a novel LMNA mutation, c.1455A $>$ G (Pro485Arg) in exon 8, that was identified in a U.S. Caucasian patient referred for a molecular diagnosis of Werner syndrome. Her clinical presentation was consistent with a type of partial lipodystrophy as well as the Werner syndrome.

The presence of nuclear morphological abnormalities characteristic of cells derived from laminopathy patients supports the likelihood that the Pro485Arg change is responsible for the disorder. We observed nuclei with irregular morphologies in primary fibroblasts but not in a lymphoblastoid cell line derived from the patient. As noted earlier [Nguyen et al., 2007], immortalization appeared to mask the nuclear morphological abnormalities of LMNA mutant cells, at least for the case of less severe types of laminopathies. This is consistent with the finding that lamin A expression levels are dependent on the state of cellular differentiation as well as with observations that lamin A expression is reduced in cancer cells [Oguchi et al., 2002].

According to the diagnostic criteria for Werner syndrome as delineated in our Registry (http://www.pathology.washington.edu/research/werner/registry/registry. $\mathrm{html}$ ), this patient met criteria for possible WS because of her skin changes, short stature, premature thinning of hair, hypogonadism, and voice changes. If she had bilateral ocular cataracts, she would have had 3 cardinal features (short stature, skin changes and cataracts) and thus would have met the criteria for probable Werner syndrome. If, in addition to cataracts, she had an affected sibling, or her parents were consanguineous, she would have met diagnostic criteria for definite Werner syndrome (all cardinal signs plus 2 further signs). The presence of bilateral ocular cataracts by age 30 years is one of the critical diagnostic signs of Werner syndrome. It has been reported in virtually all cases shown to have $W R N$ mutations at the time of referral [Huang et al., 2006]. This patient also lacked some key features of FPLD, diabetes mellitus and hyperlipidemia.

Mutations in LMNA are associated with a wide variety of genetic conditions besides Dunnigan type familial partial lipodystrophy, including autosomal dominant EmeryDreifuss muscular dystrophy (characterized by skeletal muscle atrophy and weakness, joint contractures, cardiac conduction abnormalities and cardiomyopathy, dilated cardiomyopathy with conduction-system disease), autosomal recessive mandibuloacral dysplasia (characterized by postnatal growth deficiency, mandibular hypoplasia, beak-like nose, short contracted fingers, acro-osteolysis, thin, mottled skin, and abnormal fat distribution) and the Hutchinson-Gilford progeria syndrome, a sporadic, early childhood segmental progeroid syndrome, sometimes referred to as 'Progeria of Childhood', to differentiate it from the Werner syndrome, which is sometimes referred to as 'Progeria of the Adult'. A series of LMNA mutations have also been reported in less well-characterized atypical progeroid syndromes [Garg et al., 2009]. Since a number of progeroid features seen in Werner syndrome are also 
seen in patients with laminopathies (table 2), a range of LMNA mutations are potential causes of atypical Werner syndrome [Chen et al., 2003].

There remains a formal possibility that our patient harbors mutations in other loci that have caused her lipodystrophic and/or progeroid features. However, the above findings collectively support the notion that the Pro485Arg change found in LMNA in subject VGS1010 is primarily responsible for her clinical features.

No mutation was identified in the ZMPSTE24 gene in this patient.

\section{Acknowledgements}

We thank Ms. Michelle K. Drews for the editorial assistance. This work was supported by R24CA78088 (G.M.M.), R21AG033313 (J.O.), the Ellison Medical Foundation Senior Scholar Award (J.O.), and the German Research Foundation (DFG) in the framework of the Cologne Excellence Cluster on Cellular Stress Responses in Aging-Associated Diseases (C.K.).

\section{References}

Broers JL, Ramaekers FC, Bonne G, Yaou RB, Hutchison CJ: Nuclear lamins: laminopathies and their role in premature ageing. Physiol Rev 86:967-1008 (2006).

-Cao H, Hegele RA: Nuclear lamin A/C R482Q mutation in Canadian kindreds with Dunnigan-type familial partial lipodystrophy. Hum Mol Genet 9:109-112 (2000).

Chen L, Lee L, Kudlow BA, Dos Santos HG, Sletvold $\mathrm{O}$, et al: $L M N A$ mutations in atypical Werner's syndrome. Lancet 362:440-445 (2003).

Dunnigan MG, Cochrane MA, Kelly A, Scott JW: Familial lipoatrophic diabetes with dominant transmission. A new syndrome. Q J Med 43:33-48 (1974).

-Fisher DZ, Chaudhary N, Blobel G: cDNA sequencing of nuclear lamins $\mathrm{A}$ and $\mathrm{C}$ reveals primary and secondary structural homology to intermediate filament proteins. Proc Natl Acad Sci USA 83:6450-6454 (1986).

-Garg A: Gender differences in the prevalence of metabolic complications in familial partial lipodystrophy (Dunnigan variety). J Clin Endocrinol Metab 85:1776-1782 (2000).

-Garg A: Acquired and inherited lipodystrophies. N Engl J Med 350:1220-1234 (2004).

- Garg A, Subramanyam L, Agarwal AK, Simha V, Levine B, et al: Atypical progeroid syndrome due to heterozygous missense LMNA mutations. J Clin Endocrinol Metab 94:49714983 (2009).

-Haque WA, Oral EA, Dietz K, Bowcock AM, Agarwal AK, et al: Risk factors for diabetes in familial partial lipodystrophy, Dunnigan variety. Diabetes Care 26:1350-1355 (2003).
Hegele R: LMNA mutation position predicts organ system involvement in laminopathies. Clin Genet 68:31-34 (2005).

Hegele RA, Oshima J: Phenomics and lamins: from disease to therapy. Exp Cell Res 313: 2134-2143 (2007).

Huang S, Kennedy BK, Oshima J: LMNA mutations in progeroid syndromes. Novartis Found Symp 264:197-202; discussion 202207, 227-230 (2005).

Huang S, Lee L, Hanson NB, Lenaerts C, Hoehn $\mathrm{H}$, et al: The spectrum of WRN mutations in Werner syndrome patients. Hum Mutat 27: 558-567 (2006).

-Huang S, Risques RA, Martin GM, Rabinovitch PS, Oshima J: Accelerated telomere shortening and replicative senescence in human fibroblasts overexpressing mutant and wildtype lamin A. Exp Cell Res 314:82-91 (2008).

Lanktree M, Cao H, Rabkin SW, Hanna A, Hegele RA: Novel LMNA mutations seen in patients with familial partial lipodystrophy subtype 2 (FPLD2; MIM 151660). Clin Genet 71:183-186 (2007).

$\checkmark$ Nguyen D, Leistritz DF, Turner L, MacGregor D, Ohson K, et al: Collagen expression in fibroblasts with a novel LMNA mutation. Biochem Biophys Res Commun 352:603-608 (2007).

Oguchi M, Sagara J, Matsumoto K, Saida T, Taniguchi S: Expression of lamins depends on epidermal differentiation and transformation. Br J Dermatol 147:853-858 (2002).

Risques RA, Arbeev KG, Yashin AI, Ukraintseva SV, Martin GM, et al: Leukocyte telomere length is associated with disability in older U.S. population. J Am Geriatr Soc 58:12891298 (2010).
Shackleton S, Lloyd DJ, Jackson SN, Evans R, Niermeijer MF, et al: $L M N A$, encoding lamin $\mathrm{A} / \mathrm{C}$, is mutated in partial lipodystrophy. Nat Genet 24:153-156 (2000).

Speckman RA, Garg A, Du F, Bennett L, Veile R, et al: Mutational and haplotype analyses of families with familial partial lipodystrophy (Dunnigan variety) reveal recurrent missense mutations in the globular C-terminal domain of lamin A/C. Am J Hum Genet 66: 1192-1198 (2000).

Subramanyam L, Simha V, Garg A: Overlapping syndrome with familial partial lipodystrophy, Dunnigan variety and cardiomyopathy due to amino-terminal heterozygous missense lamin A/C mutations. Clin Genet 78: 66-73 (2010).

Van der Kooi AJ, Bonne G, Eymard B, Duboc D, Talim B, et al: Lamin $A / C$ mutations with lipodystrophy, cardiac abnormalities, and muscular dystrophy. Neurology 59:620-623 (2002).

Vantyghem MC, Vincent-Desplanques D, Defrance-Faivre F, Capeau J, Fermon C, et al: Fertility and obstetrical complications in women with $L M N A$-related familial partial lipodystrophy. J Clin Endocrinol Metab 93: 2223-2229 (2008)

Vigouroux C, Auclair M, Dubosclard E, Pouchelet M, Capeau J, et al: Nuclear envelope disorganization in fibroblasts from lipodystrophic patients with heterozygous R482Q/W mutations in the lamin $A / C$ gene. J Cell Sci 114:4459-4468 (2001).

Worman HJ, Bonne G: 'Laminopathies': a wide spectrum of human diseases. Exp Cell Res 313:2121-2133 (2007). 